\title{
Chemokine receptor 7 promotes tumor migration and invasiveness via the RhoA/ROCK pathway in metastatic squamous cell carcinoma of the head and neck
}

\author{
ZHONGFEI XU ${ }^{1,2}$, XIAOJIAO ZHENG ${ }^{1,2}$, LIANGLIANG YANG ${ }^{3}$, FAYU LIU $^{1,2}$, ENJIAO ZHANG ${ }^{1,2}$, \\ WEIYI DUAN ${ }^{1,2}$, SHUANG BAI ${ }^{1,2}$, JAWAD SAFDAR ${ }^{1,2}$, ZHENNING LI $^{1,2}$ and CHANGFU SUN ${ }^{1,2}$ \\ ${ }^{1}$ Department of Oromaxillofacial-Head and Neck Surgery, School of Stomatology, China Medical University; \\ ${ }^{2}$ Department of Oral Maxillofacial Surgery, School of Stomatology, China Medical University; ${ }^{3}$ Department of Stomatology, \\ The Fourth Affiliated Hospital of China Medical University, Shenyang, Liaoning 110002, P.R. China
}

Received September 24, 2014; Accepted November 13, 2014

DOI: 10.3892/or.2014.3631

\begin{abstract}
Metastatic squamous cell carcinoma of the head and neck (SCCHN) has been shown to express chemokine receptor 7 (CCR7), which can activate signaling pathways to promote invasion and survival of SCCHN cells. We hypothesized that the RhoA/Rho-associated kinase (ROCK) pathway is involved in the CCR7-induced invasion and migration of metastatic SCCHN cells. Thus, using migration, matrigel invasion and scrape wound-healing assays, we elucidated the role of RhoA in mediating CCR7-associated cellular mobility. Pull-down assays and western blotting were used to measure RhoA and its downstream expression. Immunohistochemical staining and analysis were useful in identifying the correlation between CCR7 and RhoA expression and clinicopathological factors. The results showed that inhibition of RhoA/ROCK reduced the tumor cell migration and invasiveness induced by CCL19. Activated RhoA, proline-rich tyrosine kinase-2 (Pyk2) and cofilin induced by CCL19 were elevated, and increased RhoA, Pyk 2 and cofilin activity was eliminated by CCR $7 m A b$, RhoA/ROCK and Pyk2 inhibitors, indicating involvement of the RhoA/ROCK-Pyk2-cofilin cascade. In summary, CCR7 via RhoA/ROCK-Pyk2 cofilin pathway promotes invasion and migration of metastatic SCCHN cells.
\end{abstract}

\section{Introduction}

Squamous cell carcinoma of the head and neck (SCCHN) accounts for over $90 \%$ of all head and neck cancer (1). The

Correspondence to: Dr Changfu Sun, Department of Oral Maxillofacial Surgery, School of Stomatology, China Medical University, No. 117 Nanjing North Street, Heping, Shenyang, Liaoning 110002, P.R. China

E-mail: changfusun@hotmail.com; xzf7090@hotmail.com

Key words: RhoA, ROCK, CCR7, Pyk2, cofilin, squamous cell carcinoma of head and neck 5-year survival for patients is only $30-40 \%$, mainly due to lymphatic metastatic and local recurrence (2). No significant increase in the long-term survival rate has been achieved during the past 30 years, despite substantial improvements in the surgical management of radiotherapy and chemotherapy for SCCHN. The mechanisms determining the directional migration and invasion of SCCHN cells into specific organs remain to be elucidated.

Chemokines induce cytoskeleton rearrangement, firm adhesion to endothelial cells, and directional migration by binding to G-protein-coupled receptors (3). Chemokines and their respective receptors were involved in the metastasis of several types of cancer (4-8). The metastatic SCCHN cells were shown to express chemokine receptor 7 (CCR7), which activated phosphoinositide-3 kinase (PI3K) Cdc42, Pyk2, mTOR and NF- $\mathrm{B}$ pathways to promote 'homing' into lymph nodes and support their survival (9-12). However, when these downstream molecules were inhibited, the effect of CCR7 was not blocked completely, revealing the involvement of other downstream signaling molecules in the process.

Attention has focused on the involvement of Rho family GTPases and their downstream effectors in chemokineelicited migration (13-15). Important Rho effectors are the serine/threonine kinase Rho-associated kinase (ROCK) (16). ROCKs are important Rho effectors that enhance myosin light chain (MLC) and cofilin phosphorylation, thus regulating actin-myosin contraction (17) and cell motility (18). Pyk2 is another mediator of chemokine receptors. It has been reported that the Rho/Pyk2/cofilin pathway controls chemotaxis and the migration speed of dendritic cells (14).

In the present study we examined whether RhoA was activated by CCR7, as well as the role and the molecular mechanisms of the RhoA pathway in CCR7 regulating SCCHN metastasis. The results showed that CCL19 induced activation of the Rho/ROCK-Pyk2-cofilin pathway, whereas MLC was not involved in the process. Since chemical inhibitors of this signal transduction pathway are currently in use clinically (19), our results suggested that the ROCK inhibitor Y-27632 may be useful for treating SCCHN patients. 


\section{Materials and methods}

Cell line and human tumor samples. The PCI-37B metastatic SCCHN cell line, which strongly expresses CCR7, was a kind gift from the University of Pittsburgh (12). Cells were cultured in Dulbecco's modified Eagle's medium (DMEM) (Invitrogen, Carlsbad, CA, USA), which contained $10 \%$ fetal bovine serum (Gibco-BRL Corporation, Grand Island, NY, USA), $100 \mathrm{U} / \mathrm{ml}$ penicillin and $100 \mathrm{U} / \mathrm{ml}$ streptomycin. When inhibitors were used, treatment at the dose used did not affect cell viability or CCR7 expression.

SCCHN tissue specimens were obtained from 75 patients by biopsy prior to chemotherapy or radiotherapy at the Department of Oral and Maxillofacial Surgery, School of Stomatology, China Medical University. The term 'metastatic' in the present study refers to patients with positive lymph nodes that were recognized at initial presentation or later based on histopathological diagnosis following neck dissection. The classification of SCCHN, including primary tumors $(\mathrm{T})$, regional lymph nodes $(\mathrm{N})$, distant metastasis $(\mathrm{M})$ and stage grouping, was determined according to the regulations of the Union for International Cancer Control (UICC) for Head and Neck Cancer [tumor-node-metastasis (TNM) classification, 1997]. Ten samples of normal tissues adjacent to the benign tumor were chosen as controls. The study protocol was approved by the Medical Ethics Committee of the Affiliated Stomatological Hospital of China Medical University and performed according to the declaration of Helsinki. All the specimens were obtained with the consent of the patients prior to surgery and in accordance with the Health Insurance Portability. Written informed consent was obtained from all individuals.

Reagents and antibodies. The CCR7 chemokine ligand, CCL19 (MIP-3 $\beta$ ) was purchased from R\&D Systems (Minneapolis, MN, USA). Mouse anti-CCR7 antibody was purchased from BD Biosciences (San Jose, CA, USA). C3 exoenzyme (Rho inhibitor) was purchased from Cytoskeleton Inc. (Denver, CO, USA) and Y-27632 (ROCK inhibitor) was purchased from Sigma (Santa Clara, CA, USA). Tyrphostin A9 (Pyk2 inhibitor) was purchased from Calbiochem (San Diego, CA, USA). Anti-RhoA, anti-phospho-Pyk2 (Tyr402), anti-phospho-cofilin (Ser3) and anti-phospho-myosin light chain (Ser19) were purchased from Santa Cruz Biotechnology, Inc. (Santa Cruz, CA, USA). Bio-Rad protein assay dye reagent was purchased from Bio-Rad Laboratories (Richmond, CA, USA). Enhanced chemiluminescence was purchased from Amersham Pharmacia Biotechnology (Piscataway, NJ, USA).

Immunohistochemical analysis. Immunohistochemical staining used conventional horseradish peroxidase immunohistochemical staining methods. Briefly, $5-\mu \mathrm{m}$ sections of the specimens were deparaffinized and hydrated with $0.6 \% \mathrm{H}_{2} \mathrm{O}_{2}$ in methanol to inhibit endogenous peroxidase. Antigen retrieval was performed and slides were incubated with normal blocking serum for $10 \mathrm{~min}$. Sections were incubated with primary antibodies (1:100 dilution): CCR7-specific monoclonal antibody, and rabbit anti-RhoA polyclonal antibody overnight at $4^{\circ} \mathrm{C}$. Immunodetection was performed using peroxidase-labeled secondary antibody (R\&D Systems) and diaminobenzidine for visualization. The sections were counterstained with hematoxylin (Sigma). Negative controls included omission of the primary antibody. Cell morphology was analyzed by microscopy (Nikon Eclipse 80i; Tokyo, Japan) at x100-400 magnification. According to the percentage of positive tumor cells, the cells were scored as negative $(-),<10 \%$ or no staining; weak positive $(+), 11-50 \%$; positive $(++), 51-75 \%$; or strongly positive (+++), $>75 \%$.

Chemotaxis assay. Chemotaxis in response to chemokines was measured by counting cells migrating through a polycarbonate filter (8- $\mu \mathrm{m}$ pore size) in 24-well Transwell chambers in triplicate in DMEM with 0.5\% (w/v) BSA (Invitrogen). Cell suspensions $\left(2 \times 10^{5}\right.$ cells $\left./ 200 \mu \mathrm{l}\right)$ were placed in the top chamber of the filter. Aliquots of chemokines were added to wells. After $24 \mathrm{~h}$, the cells in each lower well were counted under a light microscope in at least five different fields (original magnification, $\mathrm{x} 200$ ). Means $\pm \mathrm{SD}$ were recorded for each condition, and an index was calculated based on the control involving random migration.

Matrigel invasion assay. Cell invasion was quantified in vitro using matrigel-coated semipermeable, modified inserts with a pore size of $8-\mu \mathrm{m}$. Analysis of the invasion assay was performed as described in the chemotaxis assay incubated with CCL19 for $36 \mathrm{~h}$. An invasion index was normalized to non-specific cell invasion (from media-pulsed wells).

Scrape wound-healing assay. Migration of PCI-37B cells was measured using an in vitro monolayer wound assay (20). Cells grown in 24-well plates in DMEM to confluence were scraped with a pipette tip to create a cell-free area. Wounded monolayers were washed with serum-free media. The cells were pretreated with/without RhoA inhibitor C3 exoenzyme (50 ng/ml), Y-27632 ROCK inhibitor $(10 \mathrm{M})$ and CCL19 $(200 \mathrm{ng} / \mathrm{ml})$ for $1 \mathrm{~h}$. Wound closure was followed after 0 and $24 \mathrm{~h}$. The wound-healing effect was calculated as the percentage of the remaining cell-free area compared to the initial wound area (arbitrarily set as 100\%). Monolayer wound assays included serum-free DMEM controls. The wounds were observed by phase contrast microscopy (Nikon TE2000-S Eclipse) at x200 magnification and images captured were documented with photography. Each experiment was performed in triplicate, analyzing four or five scratches per well. The relative cell-free area was calculated based on the control group.

RhoA pull-down assay. RhoA-GTP was measured with recombinant purified glutathione-S-transferase rhotekin Rho-binding domain (GST-TRBD) bound to glutathione beads and purified as previously described (21). SCCHN cells were stimulated with CCL19 in serum-free medium lysed with ice-cold RIPA buffer and cleared by centrifugation. SCCHN cell lysates were incubated for $1 \mathrm{~h}$ at $4^{\circ} \mathrm{C}$ with GST or GST-TRBD coupled to glutathione-sepharose beads. Beads were then washed and boiled in SDS sample buffer and precipitates were analyzed with immunoblotting using an anti-RhoA antibody and then developed using chemiluminescence.

Western blotting. Cells were harvested in a lysis buffer $(10 \mathrm{mM}$ Tris-HCl, pH 7.6, $50 \mathrm{mM} \mathrm{NaF}, 1 \mathrm{mM} \mathrm{NaV}_{3} \mathrm{O}_{4}, 1 \%$ Triton X-100 

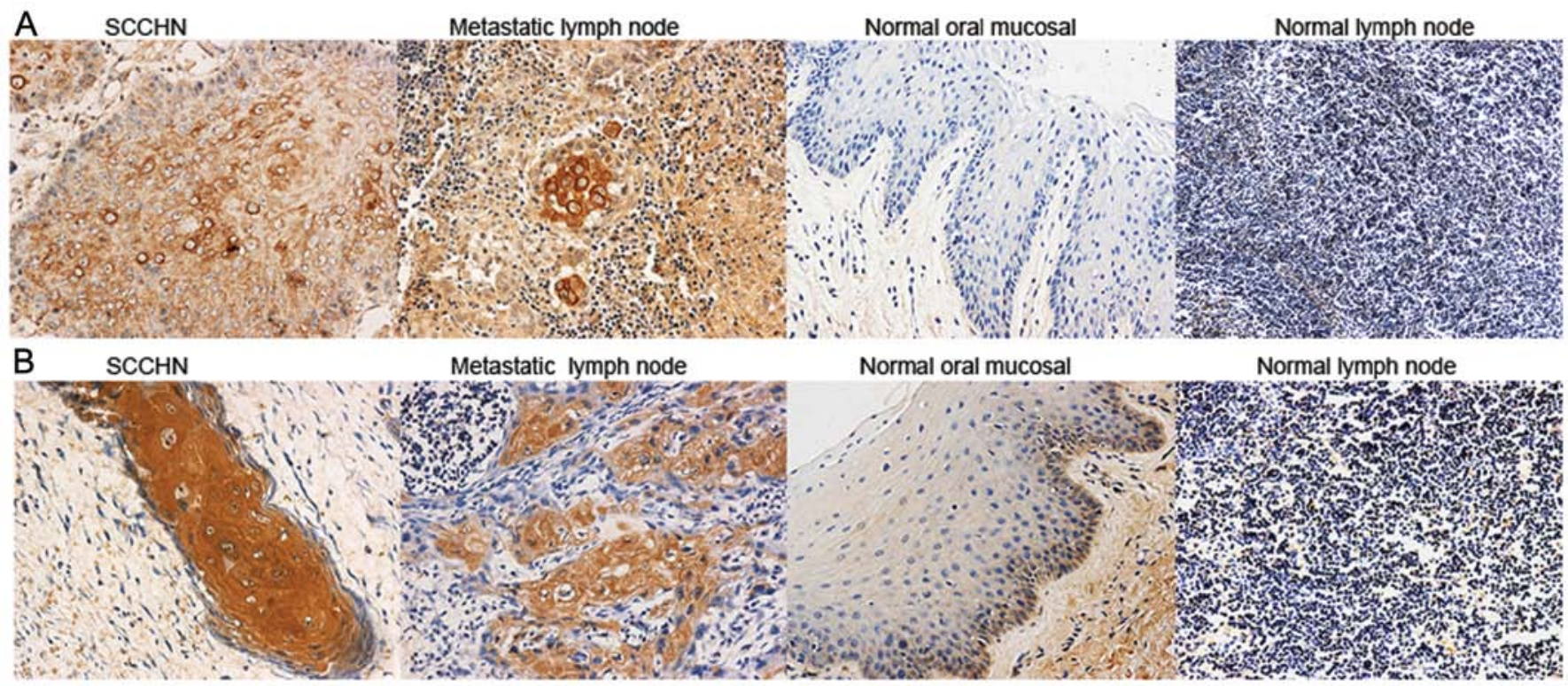

Figure 1. Immunohistochemical staining of CCR7 and RhoA in normal tissues, primary SCCHN and metastatic lymph nodes, normal lymph nodes and normal human oral mucosal tissues (original magnification, x200) indicate immunoreactivity for (A) CCR7 and (B) RhoA. CCR7 immunoreactivity was observed mainly in the cell membrane and cytoplasm of tumor cells, conforming to RhoA immunoreactivity. CCR7 and RhoA expressed in normal lymph nodes and normal human oral mucosal tissues were weak or absent. CCR7, chemokine receptor 7; SCCHN, squamous cell carcinoma of the head and neck.

and $1 \mathrm{X}$ protease inhibitor of protein tyrosine phosphatases). Lysates were sonicated for $3 \mathrm{sec}$ and centrifuged at $4^{\circ} \mathrm{C}$ at $12,000 \mathrm{rpm}$ for $30 \mathrm{~min}$. The supernatant was collected for protein quantification using the Bio-Rad protein assay dye reagent. Protein $(50 \mathrm{mg})$ was size-fractionated through a $10 \%$ SDS-PAGE gel and transferred onto nitrocellulose filters which were blocked ( $1 \%$ non-fat dry milk, $0.1 \%$ Triton X-100, $150 \mathrm{mM} \mathrm{NaCl}, 50 \mathrm{mM}$ Tris, $\mathrm{pH} \mathrm{7.5)}$ and incubated with primary antibody (1:1,000 dilution). Nitrocellulose filters were incubated with horseradish peroxidase-conjugated secondary antibodies and bands were visualized with enhanced chemiluminescence and quantified by scanning densitometry using ImageJ software.

Statistical analysis. Experiments were run in triplicate and repeated at least three times. Numerical data were expressed as means \pm standard deviation (SD). Correlations were analyzed using the Spearman's and $\chi^{2}$ tests. Statistical differences between two groups were evaluated using an unpaired Student's t-test. $\mathrm{P}<0.05$ was considered to indicate a statistically significant result. Statistical analyses were performed with SPSS 13.0 software.

\section{Results}

CCR7 and RhoA are overexpressed and CCR7 and RhoA expression is significantly positively correlated in SCCHN. Immunohistochemistry was utilized to examine CCR7 and RhoA expression in SCCHN tumor tissues, metastatic and normal lymph nodes and oral mucosal tissues. CCR7 and RhoA were evident in the cell membrane and cytoplasm, and were mainly expressed in the stromal surroundings of tumors and metastatic lymph node cells. Stained cells were few or absent in normal lymph nodes and oral mucosal tissues (Fig. 1). CCR7 and RhoA expression was significantly correlated with
Table I. Correlation between CCR7 expression and SCCHN clinicopathological characteristics.

\begin{tabular}{lcccc}
\hline Characteristics & Cases & +-+++ & $+(\%)$ & $\chi^{2}$ \\
\hline $\begin{array}{l}\text { Age (years) } \\
\quad 39 / 36\end{array}$ & $29 / 25$ & $74.4 / 69.4$ & 0.224 \\
Male/female & $42 / 33$ & $29 / 25$ & $69.0 / 75.8$ & 0.413 \\
Tumor size & & & & \\
T1, T2 & 24 & 16 & 66.7 & 0.498 \\
T3, T4 & 51 & 38 & 74.5 & 0.595 \\
$\quad$ High diff & 48 & 36 & 75 & \\
$\quad$ Low diff & 27 & 18 & 66.7 & \\
Clinical stage & & & & \\
I, II & 20 & 10 & 50 & $6.548^{\mathrm{a}}$ \\
III, IV & 55 & 44 & 80 & \\
Nodal metastases & & & & \\
Yes & 30 & 27 & 90 & $8.036^{\mathrm{a}}$ \\
No & 45 & 27 & 60 &
\end{tabular}

${ }^{\mathrm{a}} \mathrm{P}<0.05$, difference of CCR7 expression within clinicopathological characteristics. CCR7, chemokine receptor 7; SCCHN, squamous cell carcinoma of the head and neck; diff, difference.

cervical lymph-node metastasis and SCCHN clinical stages $(\mathrm{P}<0.05$; Tables I and II). Spearman's test indicated a significant positive correlation between the expression of CCR7 and RhoA (P<0.05; Table III).

CCL19 induces RhoA GTPase activation in PCI-37B. Since CCR7 regulates actin organization (22) and the latter can be 
Table II. Correlation between RhoA expression and SCCHN clinicopathological characteristics.

\begin{tabular}{lcccc}
\hline Characteristics & Cases & +-++ & $+(\%)$ & $\chi^{2}$ \\
\hline $\begin{array}{l}\text { Age (years) } \\
\geq 60 /<60\end{array}$ & $39 / 36$ & $22 / 24$ & $56.4 / 66.7$ & 0.830 \\
Male/female & $42 / 33$ & $29 / 17$ & $69.0 / 51.5$ & 2.395 \\
Tumor size & & & & \\
T1, T2 & 24 & 13 & 54.2 & 0.764 \\
T3, T4 & 51 & 33 & 64.7 & 0.077 \\
$\quad$ High diff & 48 & 30 & 62.5 & \\
$\quad$ Low diff & 27 & 16 & 59.3 & \\
Clinical stage & & & & \\
I, II & 20 & 6 & 30 & $11.29^{\mathrm{a}}$ \\
III, IV & 55 & 40 & 72.7 & \\
Nodal metastases & & & & \\
Yes & 30 & 24 & 80 & $7.346^{\mathrm{a}}$ \\
No & 45 & 22 & 48.9 & \\
\end{tabular}

${ }^{\mathrm{a}} \mathrm{P}<0.05$, difference of RhoA expression within clinicopathological characteristics. SCCHN, squamous cell carcinoma of the head and neck; diff, difference.

Table III. Correlations between the CCR7 and RhoA expression in SCCHN primary tumor.

\begin{tabular}{lccc}
\hline & \multicolumn{2}{c}{ RhoA } & \\
\cline { 2 - 3 } CCR7 & +-+++ & - & Total \\
\hline+-+++ & 36 & 18 & 54 \\
- & 10 & 11 & 21 \\
Total & 46 & 29 & 75 \\
\hline
\end{tabular}

CCR7, chemokine receptor 7; SCCHN, squamous cell carcinoma of the head and neck.

controlled by small GTPase Rho (16), we investigated whether CCR7 induces the activation of RhoA in an SCCHN cell line (PCI-37B). We measured activated RhoA (GTP-bound form) with Rho-binding domain (RBD) pull-down assays using the Rho effector protein, Rhotekin (23). Limited active bound RhoA was observed in non-stimulated cells, and this was increased after CCL19 stimulation. Although total RhoA remained unchanged, activated RhoA increased significantly 10 min after CCL19 treatment compared with the controls $(\mathrm{P}<0.05)$, whereas CCR7mAb, a C3-exo-enzyme, partly inhibited the CCL19-induced activation of RhoA compared with the CCL19-stimulation group ( $\mathrm{P}<0.05$; Fig. 2$)$.

RhoA/ROCK regulates CCR7-dependent migration and invasion. Using chemotaxis and matrigel invasion assay we analyzed the role of RhoA in PCI-37B migration and invasiveness in response to CCL19. The presence of CCL19 in the

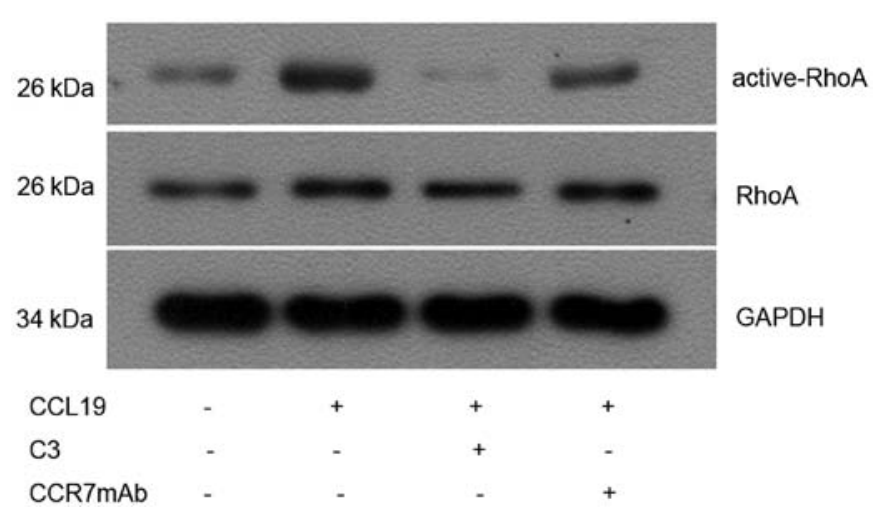

Figure 2.CCR7 stimulated the activation of RhoA. CCR7, chemokine receptor 7.

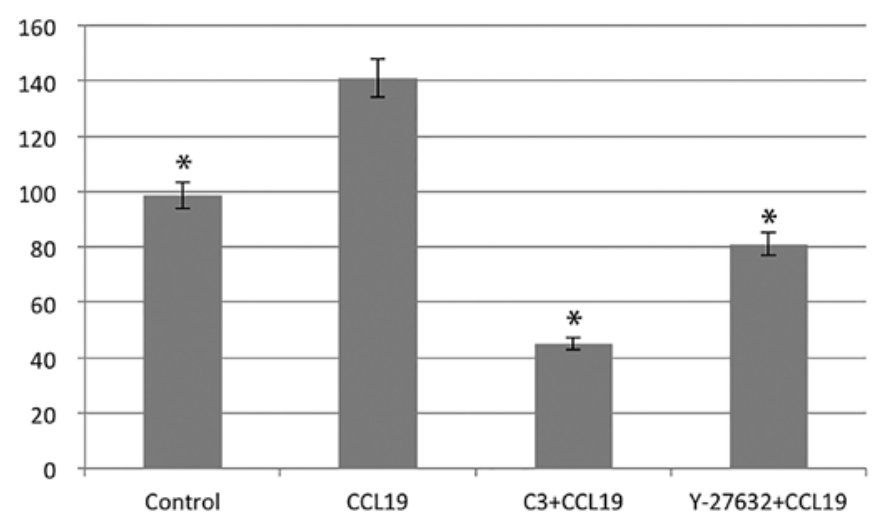

Figure 3. Role of RhoA and ROCK inhibitors in CCL19-induced cell invasion. ROCK, Rho-associated kinase.

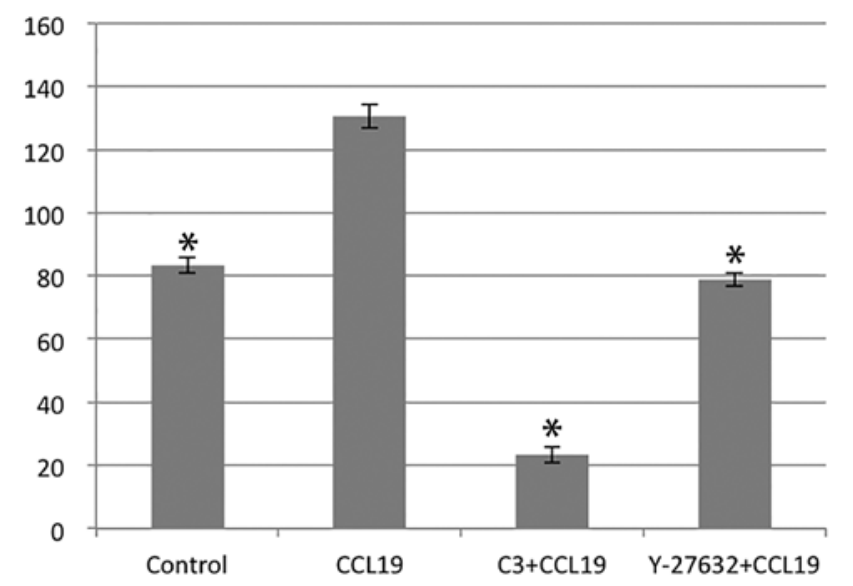

Figure 4. Role of RhoA and ROCK inhibitors after CCL19-induced cell migration. ROCK, Rho-associated kinase.

lower chamber stimulated a $>1.5$-fold increase in chemotaxis and invasion of PCI-37B across the filter membrane while $\mathrm{C} 3$ and $\mathrm{Y}-27632$ blocked this activity $(\mathrm{P}<0.05$; Figs. 3 and 4$)$. In addition, we evaluated the migration ability of PCI-37B induced by CCR7 using a scrape wound-healing assay. The width of the wound, which was significantly reduced after $24 \mathrm{~h}$ in the presence of CCL19, and this was blocked by C3 and Y-27632 (P<0.05; Fig. 5). Data show that RhoA/ROCK has a role in the invasiveness of PCI-37B mediated by CCR7. 


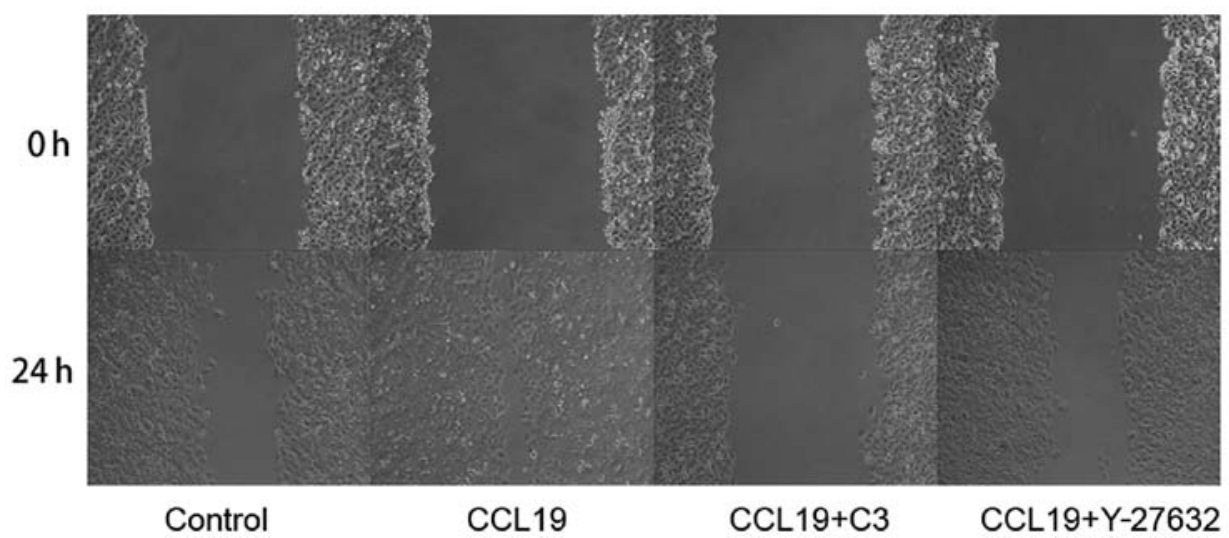

Figure 5. Roles of RhoA and ROCK inhibitors after CCL19-induced scrape wound-healing. ROCK, Rho-associated kinase.

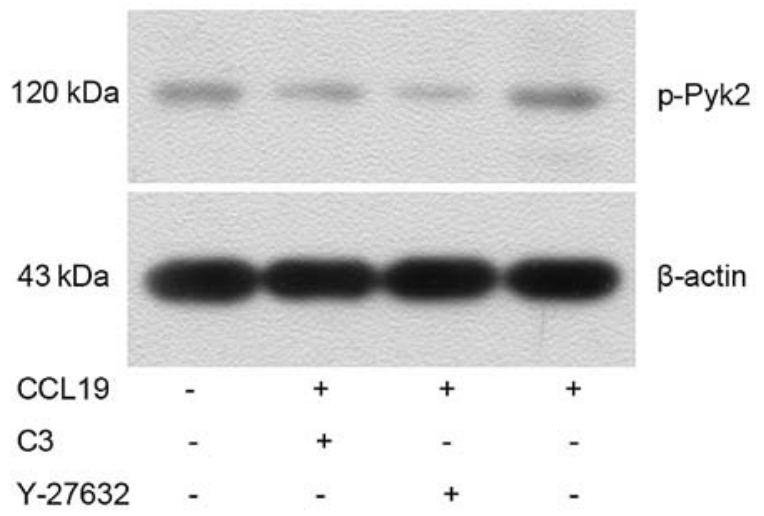

Figure 6. Western blotting shows the role of RhoA and ROCK inhibitors after CCL19-induced p-Pyk2 expression. PCI-37B cells pretreated with/without $\mathrm{C} 3(50 \mathrm{ng} / \mathrm{ml})$ or Y-27632 $\left(10 \mathrm{M}\right.$, at $37^{\circ} \mathrm{C}$ for $\left.1 \mathrm{~h}\right)$ were stimulated with CCL19 (200 ng/ml for $5 \mathrm{~min}$ ). ROCK, Rho-associated kinase.

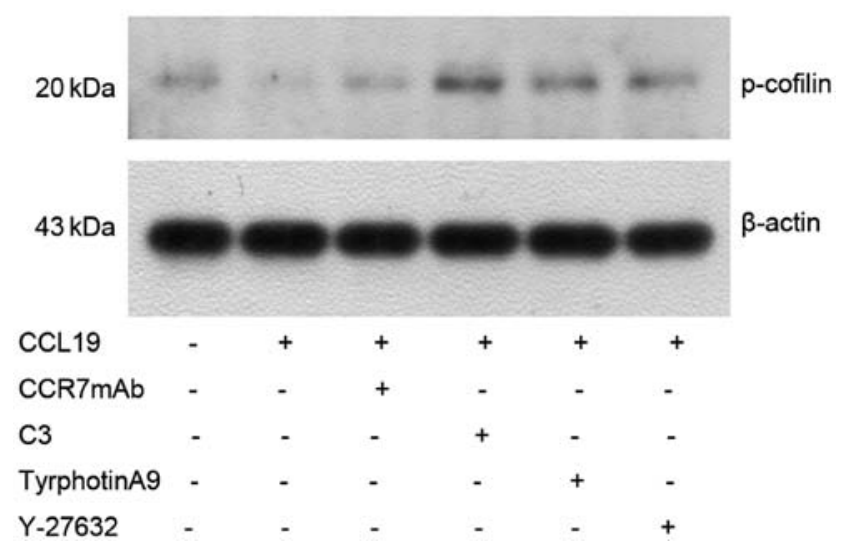

Figure 7. Western blotting shows the role of RhoA, ROCK and Pyk2 inhibitors after CCL19-induced p-cofilin expression. ROCK, Rho-associated kinase. PCI-37B cells pretreated with/without C3 $(50 \mathrm{ng} / \mathrm{ml})$ or Y-27632 $(10 \mathrm{M})$ CCR7mAb $(10 \mathrm{ng} / \mathrm{ml})$ or tyrphostin $\left(10 \mathrm{M}\right.$, at $37^{\circ} \mathrm{C}$ for $\left.1 \mathrm{~h}\right)$ were stimulated with CCL19 $(200 \mathrm{ng} / \mathrm{ml}$ for $5 \mathrm{~min})$.

RhoA/ROCK mediates the activation of Pyk 2 and cofilin induced by CCL19. Western blotting indicated that CCL19pretreated PCI-37B cells had an increased expression of p-Pyk2 (Fig. 6) and significantly decreased expression of p-cofilin (Fig. 7), which was reversed by CCR7mAb,

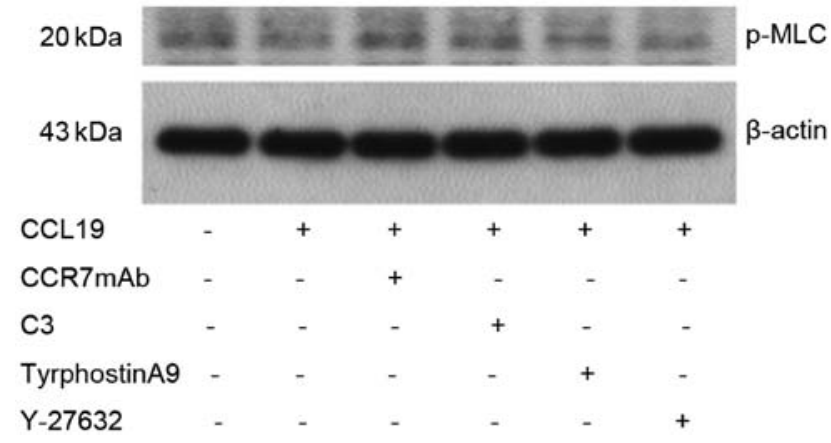

Figure 8. Western blotting shows the role of RhoA, ROCK and Pyk2 inhibitors after CCL19-induced p-MLC expression. PCI-37B cells pretreated with/ without C3 $(50 \mathrm{ng} / \mathrm{ml})$ or Y-27632 $(10 \mathrm{M})$ CCR7 mAb $(10 \mathrm{ng} / \mathrm{ml})$ or tyrphostin $\left(10 \mathrm{M}\right.$, at $37^{\circ} \mathrm{C}$ for $\left.1 \mathrm{~h}\right)$ were stimulated with CCL19 $(200 \mathrm{ng} / \mathrm{ml}$ for $5 \mathrm{~min})$. ROCK, Rho-associated kinase.

suggesting CCR7-induced activation of Pyk2 and cofilin. As expected, CCL19-dependent Pyk2 phosphorylation and cofilin dephosphorylation was abrogated in the presence of RhoA, ROCK and Pyk 2 inhibitors, suggesting that RhoA/ROCK may mediate the activation of Pyk2 and cofilin induced by CCL19. The results showed that MLC protein was not changed after CCL19 stimulation, indicating that CCR7 has no effect on MLC (Fig. 8).

\section{Discussion}

A critical problem in SCCHN therapy is metastasis, particularly to lymph nodes, lung, liver and bone. Metastasis is based on chemotaxis and the migratory ability of tumor cells. CCR7 induces chemotaxis and invasion of SCCHN cells via the activation of several signaling pathways $(9-11,24-26)$. However, how migration is influenced by the signaling pathway remains poorly understood. RhoA is also involved in regulating the migration and invasion of diverse tumor types. RiolBlanco et al revealed that CCR7 activates the Rho/Pyk2/cofilin signaling pathway, which controls chemotaxis and the migratory speed of dendritic cells (14). Other results also support a role for Rho/ROCK signaling in the regulation of CCR7dependent chronic lymphocytic leukemia migration (15). However, no reports are available to explain the role of RhoA in SCCHN. Our experiments indicate that RhoA and CCR7 are 
highly expressed in SCCHN and metastatic lymph-node cells. RhoA and CCR7 expression was significantly correlated with cervical lymph-node metastasis and SCCHN clinical stage. A Spearman's test indicated a significant positive correlation between expression of CCR7 and RhoA. Therefore, RhoA may be necessary for the development and metastasis of SCCHN, while CCR7 may regulate cell migration and survival via the RhoA pathway in SCCHN.

RhoA is upregulated in various human tumor types. RhoA stimulates cell cycle progression and cytokinesis, as well as cell migration (27) through its activity as a molecular switch cycling between an inactive guanosine diphosphate (GDP)-bound and an active guanosine triphosphate (GTP)-bound form $(28,29)$. A RhoA pull-down assay indicated that CCL19 (CCR7 ligand) was able to induce RhoA activation 1.5-folds more compared with controls in PCI-37B cell lines. The specific inhibitor of RhoA (C3 exoenzyme) decreased RhoA-GTP to one-third of baseline and CCR7mAb decreased RhoA-GTP significantly, however, the total RhoA did not change. The results show that RhoA may participate in the downstream pathway of CCR7 in SCCHN metastasis. Chemotaxis and Matrigel invasion, and scrape wound-healing assay data confirmed that CCL19-induced cell migration and invasion was $>1.5$-times greater than that of the controls and this was blocked with C3 exoenzyme. These data also confirm that RhoA may be an important downstream effector of the CCR7 pathway in SCCHN metastasis.

Rho GTPases participate in various signal transduction pathways via the activation of multiple downstream effector proteins. An important downstream effector is ROCK (27). Studies indicate that ROCK is a downstream effector mediating Rho tumor growth potential. ROCKs phosphorylate and activate LIMKs, which in turn phosphorylate and inactivate cofilin, an actin-regulatory protein $(27,30)$ that regulates the migration and invasion of cancer cells (31). ROCKs also regulate actomyosin contractility in cells by increasing the phosphorylation of MLC, which in turn stimulates myosin II to interact with and move along actin filaments (17).

Tyrosine kinase Pyk2 is involved in signaling from chemokine receptors in different cells (14). Our recent results suggest that CCR7 promotes tumor metastasis by sequential activation of Pyk 2 and cofilin followed by rearrangement of F-actin in SCCHN cells (25). However, whether RhoA participated in that pathway is unknown. Western blot results indicate that CCR7 induced the activation of Pyk2 and cofilin. Furthermore, CCL19-dependent Pyk2 phosphorylation and cofilin dephosphorylation was blocked by the RhoA and ROCK inhibitors, suggesting that RhoA/ROCK may mediate the activation of cofilin and Pyk2 induced by CCL19 in SCCHN cells. MLC protein was not changed following CCL19 stimulation, suggesting that CCR7 has no effect on MLC.

In conclusion, $\mathrm{CCR} 7$ promotes tumor migration and invasiveness via the RhoA/ROCK pathway in metastatic SCCHN. Direct inhibition of ROCK, a downstream molecule of Rho GTPase, using a pharmacological inhibitor (Y-27632) or a molecular approach (dominant-negative expression vector) can produce substantial therapeutic effects suggesting that direct targeting of the RhoA/ROCK pathway, alone or in combination with other targets, may be promising as a chemotherapeutic target (32). Fasudil (Y-27632), a potent and selective ROCK inhibitor, is relatively safe and effective for treating cardiovascular disease including cerebral and coronary vasospasm, angina, hypertension and heart failure. No serious adverse side-effects have been reported thus far (33). Future studies are needed to identify the effectiveness and safety of ROCK inhibitors, specifically for treatment of SCCHN. We found that ROCK inhibition may offer a novel therapeutic strategy for the total management of SCCHN as well as inhibiting metastases.

\section{Acknowledgements}

This study was supported by grants from the National Natural Science Foundation of China (no. 81372877), the National Young Scholars Science Foundation of China (no. 81102058), the Foundation of Education Bureau of Liaoning Province (nos. 2009A755 and L2014317), the Public Welfare Fund Project for Science of Liaoning Province (no. 2011002001), the Natural Science Foundation of Liaoning Province (no. 2014021096), and the Excellent Talent Fund Project of Higher Education of Liaoning Province (LJQ2014087).

\section{References}

1. Chambers AF, Groom AC and MacDonald IC: Dissemination and growth of cancer cells in metastatic sites. Nat Rev Cancer 2: 563-572, 2002

2. Siegel R, Naishadham D and Jemal A: Cancer statistics, 2013. CA Cancer J Clin 63: 11-30, 2013.

3. Zlotnik A and Yoshie O: Chemokines: a new classification system and their role in immunity. Immunity 12: 121-127, 2000.

4. Ben-Baruch A: Site-specific metastasis formation: chemokines as regulators of tumor cell adhesion, motility and invasion. Cell Adh Migr 3: 328-333, 2009.

5. Chen G, Chen SM, Wang X, Ding XF, Ding J and Meng LH: Inhibition of chemokine (CXC motif) ligand 12/chemokine (CXC motif) receptor 4 axis (CXCL12/CXCR4)-mediated cell migration by targeting mammalian target of rapamycin (mTOR) pathway in human gastric carcinoma cells. J Biol Chem 287: 12132-12141, 2012

6. Nannuru KC, Sharma B, Varney ML and Singh RK: Role of chemokine receptor CXCR2 expression in mammary tumor growth, angiogenesis and metastasis. J Carcinog 10: 40, 2011.

7. Hao M, Zheng J, Hou K, et al: Role of chemokine receptor CXCR7 in bladder cancer progression. Biochem Pharmacol 84: 204-214, 2012.

8. Lee YS, Choi I, Ning Y, et al: Interleukin-8 and its receptor CXCR2 in the tumour microenvironment promote colon cancer growth, progression and metastasis. Br J Cancer 106: 1833-1841, 2012.

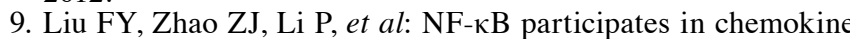
receptor 7-mediated cell survival in metastatic squamous cell carcinoma of the head and neck. Oncol Rep 25: 383-391, 2011.

10. Liu FY, Zhao ZJ, Li P, Ding X, Zong ZH and Sun CF: Mammalian target of rapamycin (mTOR) is involved in the survival of cells mediated by chemokine receptor 7 through PI3K/Akt in metastatic squamous cell carcinoma of the head and neck. Br J Oral Maxillofac Surg 48: 291-296, 2010.

11. Zhao ZJ, Liu FY, Li P, Ding X, Zong ZH and Sun CF: CCL19induced chemokine receptor 7 activates the phosphoinositide-3 kinase-mediated invasive pathway through $\mathrm{Cdc} 42$ in metastatic squamous cell carcinoma of the head and neck. Oncol Rep 25: 729-737, 2011.

12. Wang J, Xi L, Hunt JL, et al: Expression pattern of chemokine receptor 6 (CCR6) and CCR7 in squamous cell carcinoma of the head and neck identifies a novel metastatic phenotype. Cancer Res 64: 1861-1866, 2004.

13. Bardi G, Niggli V and Loetscher P: Rho kinase is required for CCR7-mediated polarization and chemotaxis of T lymphocytes. FEBS Lett 542: 79-83, 2003.

14. Riol-Blanco L, Sánchez-Sánchez N, Torres A, et al: The chemokine receptor CCR7 activates in dendritic cells two signaling modules that independently regulate chemotaxis and migratory speed. J Immunol 174: 4070-4080, 2005. 
15. Cuesta-Mateos C, López-Giral S, Alfonso-Pérez M, et al: Analysis of migratory and prosurvival pathways induced by the homeostatic chemokines CCL19 and CCL21 in B-cell chronic lymphocytic leukemia. Exp Hematol 38: 756.e4-764.e4, 2010.

16. Bishop AL and Hall A: Rho GTPases and their effector proteins. Biochem J 348: 241-255, 2000.

17. Riento K and Ridley AJ: Rocks: multifunctional kinases in cell behaviour. Nat Rev Mol Cell Biol 4: 446-456, 2003.

18. Ono S: Regulation of actin filament dynamics by actin depolymerizing factor/cofilin and actin-interacting protein 1: new blades for twisted filaments. Biochemistry 42: 13363-13370, 2003.

19. Olson MF: Applications for ROCK kinase inhibition. Curr Opin Cell Biol 20: 242-248, 2008.

20. Freitas VM, Rangel M, Bisson LF, Jaeger RG and MachadoSantelli GM: The geodiamolide $\mathrm{H}$, derived from Brazilian sponge Geodia corticostylifera, regulates actin cytoskeleton, migration and invasion of breast cancer cells cultured in three-dimensional environment. J Cell Physiol 216: 583-594, 2008.

21. Ren XD and Schwartz MA: Determination of GTP loading on Rho. Methods Enzymol 325: 264-272, 2000.

22. Muller A, Homey B, Soto H, et al: Involvement of chemokine receptors in breast cancer metastasis. Nature 410: 50-56, 2001.

23. Pellegrin S and Mellor H: Rho GTPase activation assays. In: Current Protocols in Cell Biology. Juan S. Bonifacino, et al (eds), Vol 38. John Wiley \& Sons, Inc., Hoboken, NJ, pp14.8.1-14.8.19, 2008 .
24. Wang J, Zhang X, Thomas SM, et al: Chemokine receptor 7 activates phosphoinositide-3 kinase-mediated invasive and prosurvival pathways in head and neck cancer cells independent of EGFR. Oncogene 24: 5897-5904, 2005.

25. Yang L, Liu F, Xu Z, Guo N, Zheng X and Sun C: Chemokine receptor 7 via proline-rich tyrosine kinase-2 upregulates the chemotaxis and migration ability of squamous cell carcinoma of the head and neck. Oncol Rep 28: 1659-1664, 2012.

26. Kakinuma $\mathrm{T}$ and Hwang ST: Chemokines, chemokine receptors, and cancer metastasis. J Leukoc Biol 79: 639-651, 2006.

27. Ridley AJ: RhoA, RhoB and RhoC have different roles in cancer cell migration. J Microsc 251: 242-249, 2013.

28. Etienne-Manneville S and Hall A: Rho GTPases in cell biology. Nature 420: 629-635, 2002

29. Routhier A, Astuccio M, Lahey D, et al: Pharmacological inhibition of Rho-kinase signaling with Y-27632 blocks melanoma tumor growth. Oncol Rep 23: 861-867, 2010.

30. Bernard O: Lim kinases, regulators of actin dynamics. Int J Biochem Cell Biol 39: 1071-1076, 2007.

31. Ghosh M, Song X, Mouneimne G, Sidani M, Lawrence DS and Condeelis JS: Cofilin promotes actin polymerization and defines the direction of cell motility. Science 304: 743-746, 2004.

32. Rattan R, Giri S, Singh AK and Singh I: Rho/ROCK pathway as a target of tumor therapy. J Neurosci Res 83: 243-255, 2006.

33. Shimokawa $\mathrm{H}$ and Rashid M: Development of Rho-kinase inhibitors for cardiovascular medicine. Trends Pharmacol Sci 28: 296-302, 2007. 